\title{
Multi-Direction Piezoelectric Energy Harvesting Techniques
}

\author{
Chunhua Sun ${ }^{1,2}$, Guangqing Shang ${ }^{1}$ \\ ${ }^{1}$ Department of Mechanical and Electrical Engineering, Suzhou Vocational University, Suzhou, China \\ ${ }^{2} 3 \mathrm{C}$-Product Intelligent Manufacturing Engineering Technology Research and Development Center of Suzhou, Suzhou, China \\ Email: chh_sunny@163.com
}

How to cite this paper: Sun, C.H. and Shang, G.Q. (2019) Multi-Direction Piezoelectric Energy Harvesting Techniques. Journal of Power and Energy Engineering, 7, 52-59.

https://doi.org/10.4236/jpee.2019.79003

Received: August 8, 2019

Accepted: September 16, 2019

Published: September 19, 2019

Copyright () 2019 by author(s) and Scientific Research Publishing Inc. This work is licensed under the Creative Commons Attribution International License (CC BY 4.0).

http://creativecommons.org/licenses/by/4.0/

\begin{abstract}
With the development of portable and self-powering electronic devices, micro-electromechanical system (MEMS) and wireless sensor networks, research on piezoelectric energy harvesting techniques has been paid more and more attention. To enhance the ambient adaptability and improve the generating efficiency, the multi-directional piezoelectric energy harvesting techniques turns to be a research hotspot. The current status of the multi-directional piezoelectric energy harvesting techniques was firstly reviewed. The characteristics of existed multi-directional piezoelectric harvester were then analyzed. An improved structure of multi-directional piezoelectric harvester was finally proposed. The multi-directional piezoelectric energy harvester has a good prospect in miniaturization, more sensitive to vibration directions and better energy efficiency.
\end{abstract}

\section{Keywords}

Piezoelectric Effect, Piezoelectric Energy Harvester, Multi-Directional, MEMS

\section{Introduction}

With the development of self-powering, wireless sensor networks and MEMS, researchers have concentrated a great deal of interest on harvesting wasted energy via electromagnetic, electrostatic and piezoelectric mode. Among these, the technique of piezoelectric energy harvesting has more meaningful and attracts much attention owing to its compatibility and higher energy density [1]. The number of published papers in the database of Web of Science [2] using "piezoelectric energy harvester" (abbreviated as PEH) as the key word continuously increases between 2000 and 2018 and was more than 1,267 per year in 2018, as shown in Figure 1. The different research fields of piezoelectric energy 
harvesting were also researched via the same key word, as shown in Figure 2. It can be seen that the piezoelectric energy harvesting technique has also been widely studied on application of engineering, telecommunication as well as mechanics in addition to some basic research fields.

Among these published articles, most were focused on the structure of PEH. Figure 3 shows the papers' number ratio on these typical structures of PEHs, including cantilever, cymbal and stacks, etc. It also reveals that researchers have paid much more attention to the cantilever PEHs as its simpler fabrication process and relatively larger strain.

Most of the existing cantilever PEHs are unidirectional piezoelectric devices

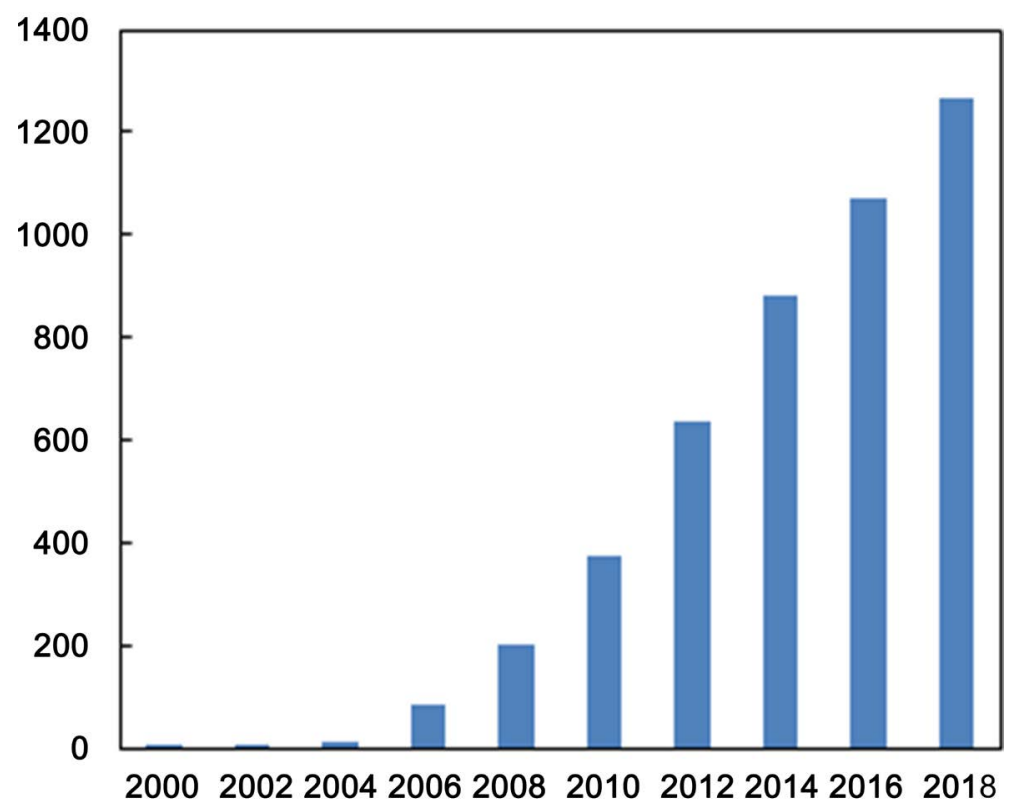

Figure 1. Published papers about PEH between 2000 and 2018.

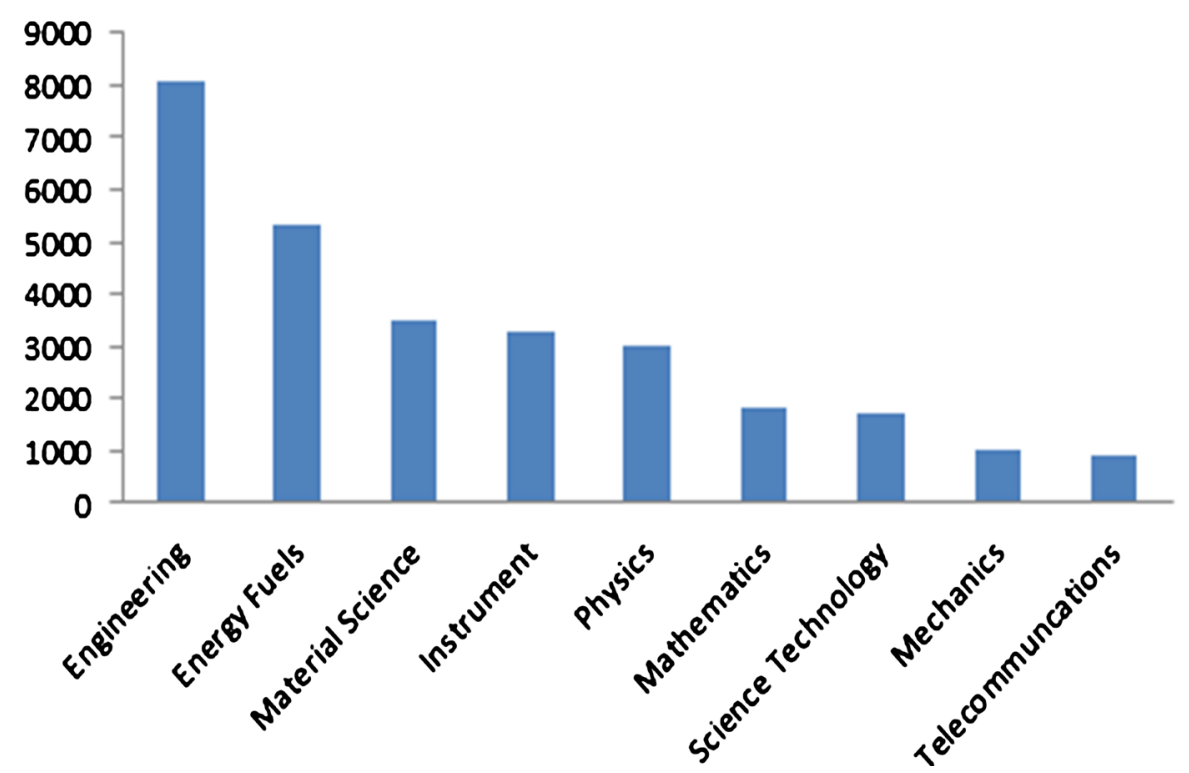

Figure 2. Published papers about PEH via research fields between 2000 and 2018. 


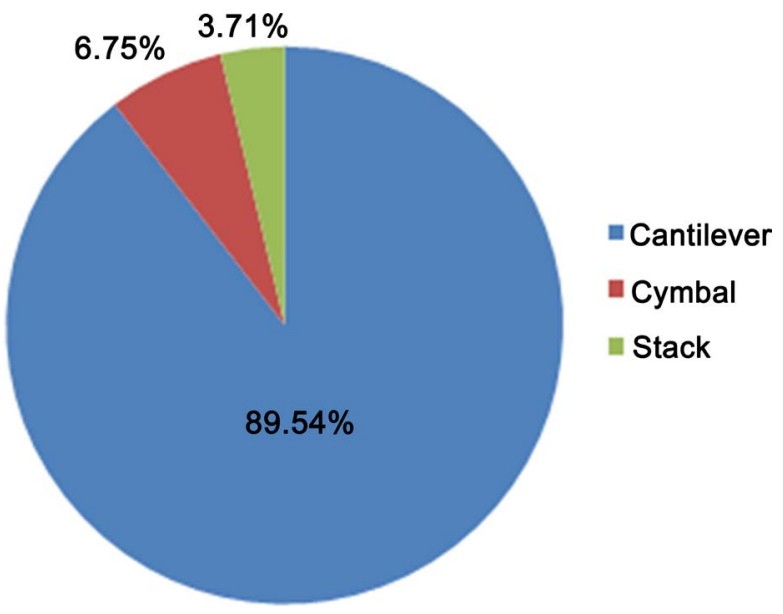

Figure 3. Published papers ratio on typical structure of PEHs [2].

based on the technology of linear vibration theory so that demand for sensitivity to vibration direction is stronger. It is very important to keep the main vibration direction of PEH be consistent with the ambient vibration. However, most of the vibration in ambient is irregular and even random. This leads to the difficulty of existing the main vibration of the designed PEH and make harvesting energy efficiency lower in the specific application.

In view of the above problems, the multi-directional piezoelectric energy harvesting techniques will be developed in this paper. The structure of the paper lists as follows: firstly, recent progress on multi-directional piezoelectric energy harvesting techniques is present. Then, characteristics of the existed multi-directional PEHs are reviewed and summarized. Based on the above analysis, a new structure of $\mathrm{PEH}$ is finally proposed to improve the harvesting efficiency and make the structure smaller and more compact.

\section{Recent Progress on Multi-Directional Piezoelectric Energy Harvesting Technique}

On improving the harvesting energy efficiency, many researches were focused on broadband harvesting techniques [3]. However, there are few papers on multi-directional PEH. From the limited literals, the conclusion can be drawn that the existing multi-directional PEHs are mainly based on cantilever beams which are correspondingly arrayed along different directions to harvest multi-directional ambient vibration.

A two-directional vibration energy harvester with radially distributed piezoelectric array [4] was proposed, as shown in Figure 4. By employing radically distributed piezoelectric array, the proposed harvester could scavenge vibration energy from various directions in a $2 \mathrm{D}$ plane. The maximum output voltage of the PEH reached $11.6 \mathrm{~V}$ by connecting the symmetric PVDF elements in series, whereas the maximum output power could obtain $13.5 \mathrm{uW}$ by connecting the symmetric PVDF elements in parallel. This proposed PEH is relatively compact and more easily to be miniaturized. However, it was difficult for the cantilever 
beam to vibrate to excite the piezoelectric effect owing to a solid cylinder, even if PVDF piezoelectric film was used as the piezoelectric material.

Using two cantilever beams to be arrayed in mutual orthogonal directions is a more commonly used method for harvesting two-directional vibration energy in ambient. A bi-directional piezoelectric energy harvester was designed by using two piezoelectric cantilevers vertical to each other, as shown in Figure 5. The influence of additional masses on electricity generation performance of PEH was studied [5].

The cube-type and dandelion-type of multi-directional PEHs were proposed [6], as shown in Figure 6. The results show that the maximum load power of cube-type harvester was $0.28 \mathrm{~mW}$ while the dandelion-type harvester was up to $0.68 \mathrm{~mW}$. Furtherly, the direction selectivity of the cube-type was weaker than the dandelion-type. Therefore, in multi-directional ambient vibration, the dandelion-type harvester was superior to the cube-type one.

A multi-directional PEH [7] was proposed by Habib A and Ananta A, as shown in Figure 7. This proposed multi-directional PEH was similar to upper half of the dandelion-type in Figure 6. Effects of key parameters of the harvester on frequency and output voltage, including mass, beam thickness and length, substrate thickness and length, were studied via the finite element method and

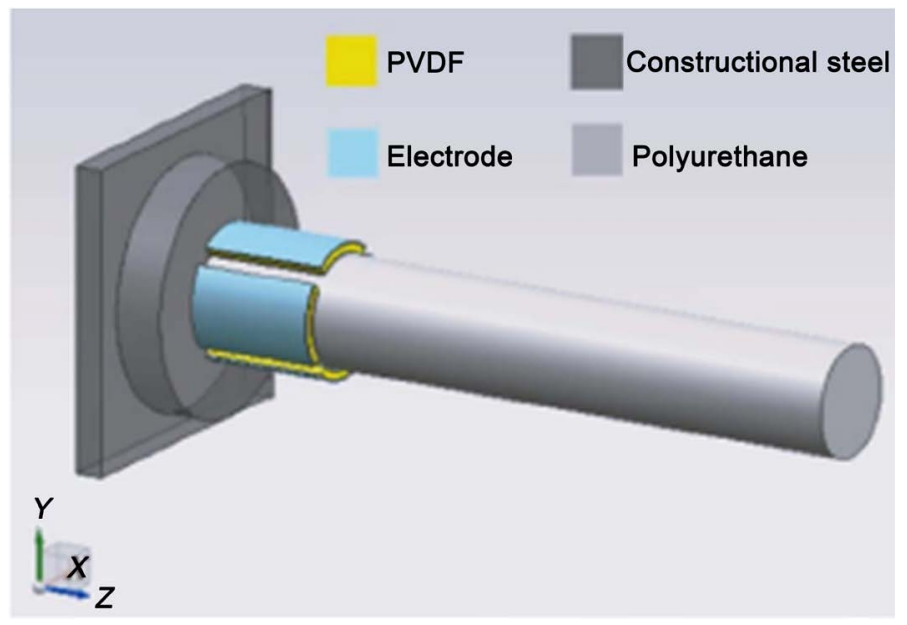

Figure 4. Illustration of radially distributed PEH [4].

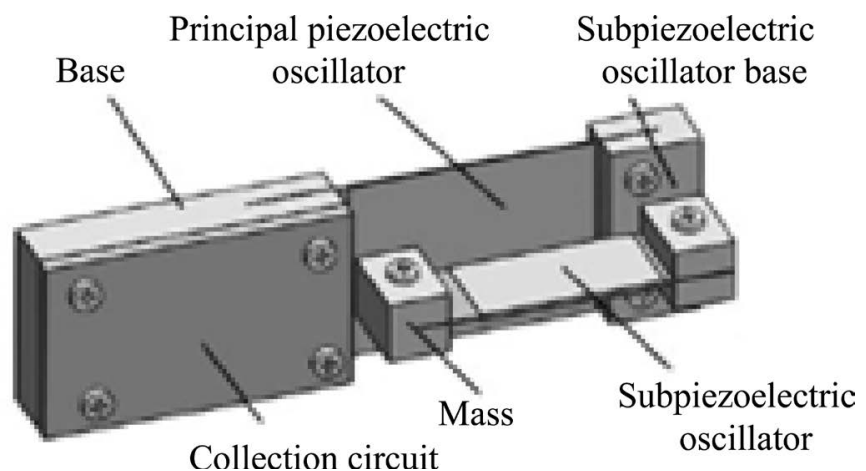

Figure 5. Schematic illustration of two-orthogonal PEH [5]. 


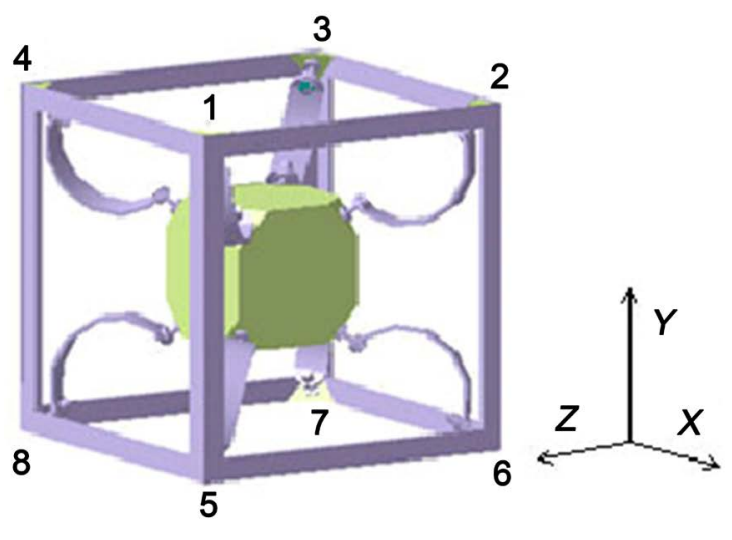

(a)

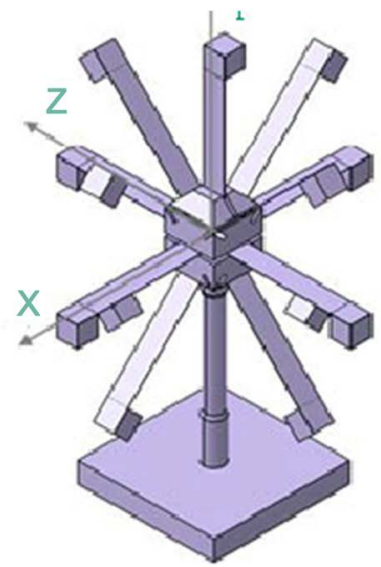

(b)

Figure 6. Two types of multi-directional PEHs [6]. (a) Cube-type; (b) Dandelion-type.

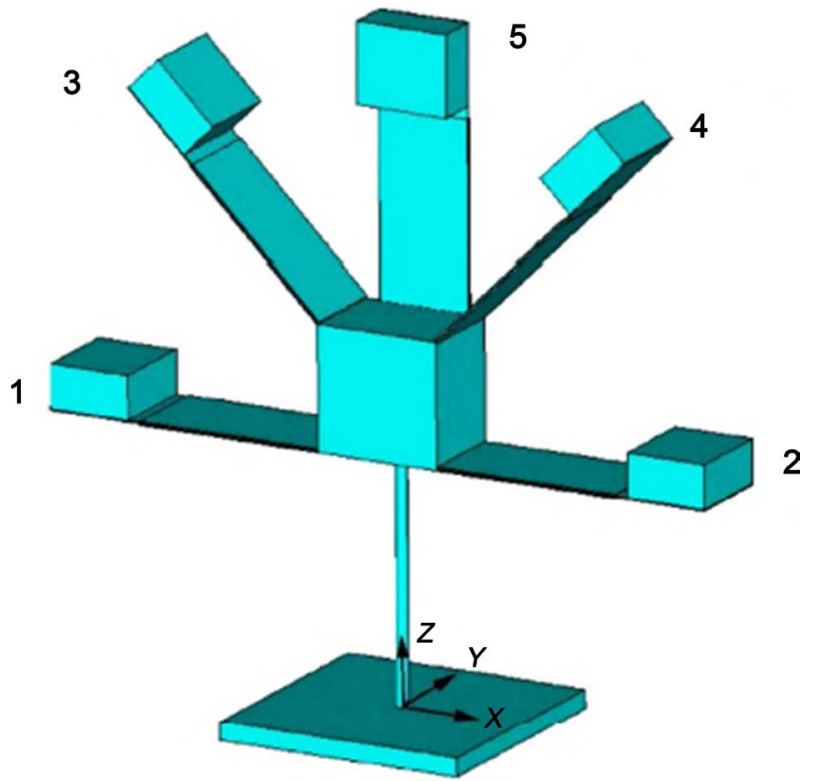

Figure 7. A multi-directional PEH [7].

experiment. Results show that the output voltage and the load power of the multidirectional vibration piezoelectric generator are larger than that of the unidirectional piezoelectric generator. The energy collection efficiency of the piezoelectric could reach the maximum when the vibration direction of the environment coincided with the piezoelectric cantilever beam. The cantilever beams with multiple directions in the piezoelectric device could largely eliminate the strong directional selectivity of the unidirectional piezoelectric generator.

In Ref. [8], a beam-roller piezoelectric energy harvester was reported to scavenge energy from both sway and bi-directional vibrations, as shown in Figure 8. The beam and the roller were sensitive to two orthogonal directions of ambient vibration. The roller was also designed to sense sway of the frame that acted as the guide rail of the roller. Design and experimental verification of this harvester showed that it was a feasible way for employing the roller to actuate 


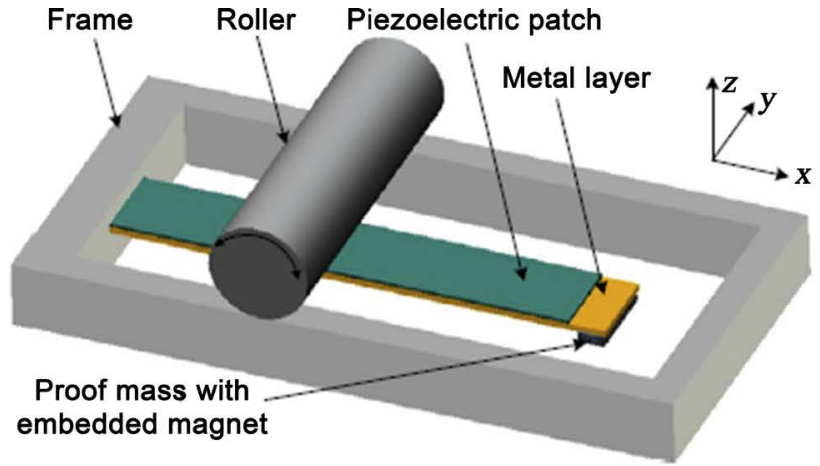

(a) Configuration of the beam-roller PEH

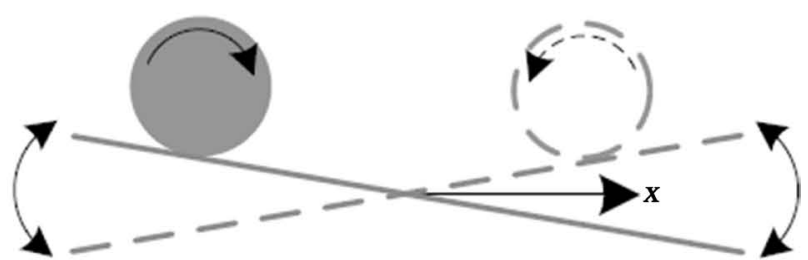

(b) Illustration of sway motion

Figure 8. Conceptual design of the beam-roller PEH [8].

the piezoelectric cantilever beam to vibrate for harvesting energy from both the sway and bi-directional vibrations. The output from the beam-roller PEH could charge a capacitor of $1 \mathrm{uF}$ through a bridge rectifier from $0 \mathrm{~V}$ to more than $4 \mathrm{~V}$ within a sway cycle. By embedding magnet within mass, this design adopts the theory of non-linear design to expand the broadband of response frequency. Meanwhile, by innovatively introducing a roller, the swing energy in a certain direction can also be collected.

\section{Analysis of the Existed Multi-Directional PEH}

From the above review, the proposed multi-directional vibration PEHs had higher energy harvesting efficiency and more sensitive to vibration directions than the traditional unidirectional piezoelectric harvester. However, the existing harvesters also have some shortcomings, which mainly embody in following:

1) Insufficient sensitivity to environmental vibration. The main reason is that the above PEHs still use some cantilever beams which are only valid for some certain frequencies and vibration directions in ambient, while most of vibration sources in ambient are random and uncertain.

2) Larger volume of the harvester. Using cantilever array has been designed and fabricated to harvest multi-directional energy from ambient in most literals. However, the pure structure array obviously enlarges the volume of the harvester, which is adverse to miniaturization of the harvester.

3) Overall inefficiency of the harvester. Most of PEHs are based on the linear vibration theory which is more effective for the vibration of a certain frequency. In fact, the ambient vibration is not only multi-directional, but also random. Therefore, the overall efficiency of PEH is lower.

So, it is necessary to innovate the design theory of multi-directional piezoelectric harvester by introducing nonlinear vibration theory to broadband vibration energy harvesting techniques.

\section{Improvement of Multi-Directional PEH}

Based on our analysis of characteristics of the existed PEHs, improvement of 


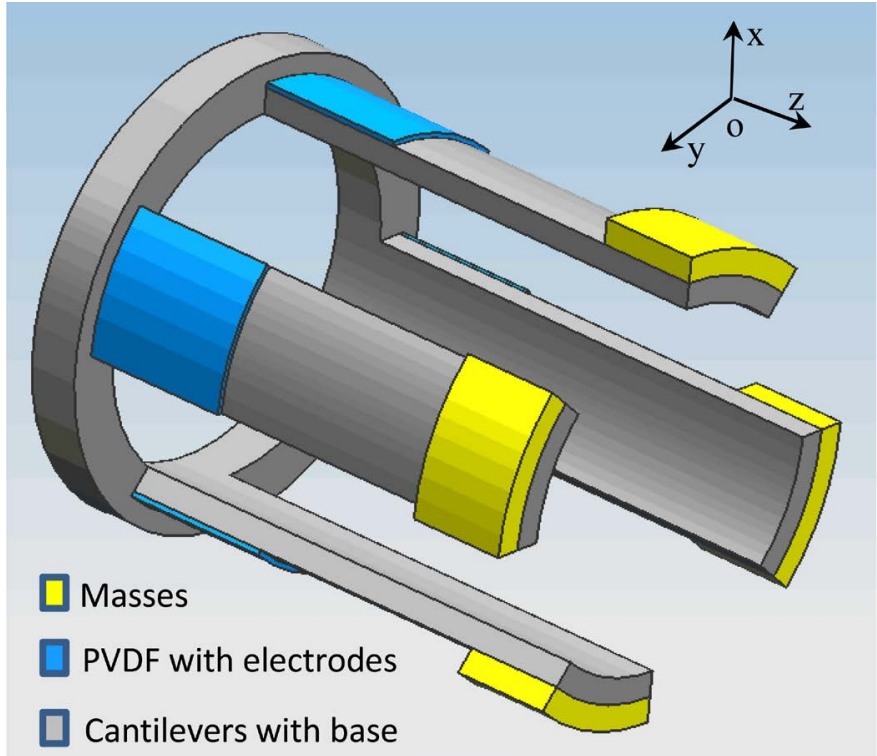

Figure 9. Improved multi-directional PEH.

$\mathrm{PEH}$ is made. Taking the multi-directional PEH in Figure 4 as an example, PVDF was used to increase the flexibility of the harvester, but the use of solid cylinder greatly limited the strain of the harvester, that will greatly reduce the sensitivity to direction and magnificent of ambient vibration and energy harvesting efficiency.

So, the improved structure in Figure 9 is proposed. Several radially symmetrical arc-shaped cantilever beams and appropriate mass blocks at the free end are adopted. With this improvement, the natural frequency of this device decreases by increasing the mass and decreasing the rigidity. The sensitivity to vibration is also greatly improved in lower frequency ambient. Therefore, this harvester can greatly improve energy harvesting efficiency and the sensitivity to vibration. Meanwhile, the structure is modified to broadband vibration with magnets as masses.

The proposed multi-directional piezoelectric vibration energy harvesting method will provide a new idea for the research of piezoelectric energy harvest. It will be helpful to the practicality and popularization of piezoelectric energy harvesting technology. It also conforms to the current trend of miniaturization of electromechanical devices. The finite element simulation and experiment will be further done to study the electromechanical performance of the improved harvester.

\section{Acknowledgements}

This work was supported by “3C-Product Intelligent Manufacturing Engineering Technology Research and Development Center of Jiangsu Province" from Jiangsu Education Department and "New Driving and Ambient Energy Harvesting Innovation Team Based on Functional Materials" from Suzhou Vocational University. 


\section{Conflicts of Interest}

The authors declare no conflicts of interest regarding the publication of this paper.

\section{References}

[1] Kim, H.S., Kim, J.H. and Kim, J. (2011) A Review of Piezoelectric Energy Harvesting Based on Vibration. International Journal of Precision Engineering and Manufacturing, 12, 1129-1141. https://doi.org/10.1007/s12541-011-0151-3

[2] Web of Science. http://wokinfo.com

[3] Evin, N. and Erturk, A. (2013) Advances in Energy Harvesting Methods. Springer, Berlin. http://www.springer.com/978-1-4614-5704-6

[4] Liu, X., Wang, P.-H., Zhang, X.-Z. and Zhao, H.-B. (2018) Two-Directional Vibration Energy Harvester with Radially Distributed Piezoelectric Array. Optics and Precision Engineering, 26, 2181-2189. https://doi.org/10.3788/OPE.20182609.2181

[5] Zhang, Q. and Niu, Q. (2018) Design and Experimental Study of Novel Bi-Directional Piezoelectric Energy Harvesting Device. Transducer and Microsystem Technologies, 37, 32-34, 38.

[6] Liu, X.J. (2012) Research on Some Key Technologies for Multi-Direction Piezoelectric Vibration Energy Harvesting. Nanjing University of Aeronautics and Astronautics, Nanjing.

[7] Habib, A. and Ananta, A. (2018) Study on Fundamental Technology of Multi Directional Vibration Piezoelectric Power Generation. World Journal of Engineering and Technology, 6, 1-16. https://doi.org/10.4236/wjet.2018.61001

[8] Fan, K.Q., Chang, J.W., Chao, F.B. and Pedrycz, W. (2015) Design and Development of a Multipurpose Piezoelectric Energy Harvester. Energy Conversion and Management, 96, 430-439. https://doi.org/10.1016/j.enconman.2015.03.014 